\title{
EXPANSIONS OF THE GROUP OF INTEGERS BY BEATTY SEQUENCES
}

\author{
AYHAN GÜNAYDIN AND MELISSA ÖZSAHAKYAN
}

\begin{abstract}
We study the model theoretic structure $\left(\mathbb{Z},+, P_{r}\right)$ where $r>1$ is an irrational number and the elements of $P_{r}$ are of the form $\lfloor n r\rfloor$ for some $n \in \mathbb{Z} \backslash\{0\}$. We axiomatize of this structure and prove a quantifier elimination result. As a consequence, we get that definable subsets are not sparse unless they are finite. We also prove that there are no reducts of this structure expanding $(\mathbb{Z},+)$.
\end{abstract}

\section{INTRODUCTION}

We investigate the expansion of the abelian group of integers by the following subset:

$$
P_{r}:=\{\lfloor n r\rfloor: n \in \mathbb{Z} \backslash\{0\}\},
$$

where $r>1$ is an irrational number. The number 0 is taken out for some technical reasons and of course it has no effect on our results. There is a considerable difference between this expansion and the expansion by $P_{r}^{+}:=P_{r} \cap \mathbb{N}$, because $\left(\mathbb{Z},+, P_{r}^{+}\right)$defines the ordering of $\mathbb{Z}$, whereas we show in Corollary 5.11 that $\left(\mathbb{Z},+, P_{r}\right)$ does not define the ordering. We also know that $P_{r}$ is not definable in $(\mathbb{Z},+,<)$, since it is shown in [3] that the only reduct of $(\mathbb{Z},+,<)$ defining addition is $(\mathbb{Z},+)$.

There have been some work on expansions of $(\mathbb{Z},+)$ by a predicate by Poizat, Palacín-Sklinos, and Lambotte-Point ([8, 7, 6]). In all these, the predicate is sparse, with certain different but similar meanings of the word sparse. Later those results were generalized by Conant (in [2]), who proved that the expansion of $(\mathbb{Z},+)$ by an infinite subset $A$ of a submonoid of $\left(\mathbb{N}_{+}, \cdot\right)$ is superstable of $U$-rank $\omega$. Hence in that setting, sparsity comes from the multiplicative structure.

Our study of the expansion $\left(\mathbb{Z},+, P_{r}\right)$ is complimentary to the work mentioned above, as the set $P_{r}$ is certainly not sparse: If $k \in P_{r}$, then the next element in $P_{r}$ is either $k+\lfloor r\rfloor$ or $k+\lfloor r\rfloor+1$. (This is also the reason of $\left(\mathbb{Z},+, P_{r}^{+}\right)$defining the ordering.) Actually, we prove that no infinite subset of $\mathbb{Z}$ definable in $\left(\mathbb{Z},+, P_{r}\right)$ is sparse in the following sense: 
Theorem 1.1. Let $X \subseteq \mathbb{Z}$ be infinite and definable in $\left(\mathbb{Z},+, P_{r}\right)$. Then there is $N=N(X) \in \mathbb{N}_{+}$such that for any $x \in X$, one of the integers $x+1, x+2, \ldots, x+N$ is in $X$.

The proof of this result goes through a quantifier elimination result. In order to state that, let $L_{ \pm}$be the extension of the language $\{+,-, 0,1\}$ of abelian groups with a distinguished element by unary predicate symbols $D_{m,+}$ and $D_{m,-}$ for each $m \geq 1$. We interpret the new symbols in $\mathbb{Z}$ as follows:

$$
D_{m,+}^{\mathbb{Z}}:=\left\{x \in \mathbb{Z}: x=m y \text { for some } y \in P_{r}\right\},
$$

and

$$
D_{m,-}^{\mathbb{Z}}:=\left\{x \in \mathbb{Z}: x=m y \text { for some } y \notin P_{r}\right\} .
$$

Note that $D_{1,+}^{\mathbb{Z}}=P_{r}$ and that $D_{m,+}^{\mathbb{Z}} \cup D_{m,-}^{\mathbb{Z}}=m \mathbb{Z}$.

Theorem 1.2. The structure $\left(\mathbb{Z},+, 0,1,\left(D_{m,+}^{\mathbb{Z}}\right)_{m \geq 1},\left(D_{m,-}^{\mathbb{Z}}\right)_{m \geq 1}\right)$ has quantifier elimination.

A particular formula with quantifiers is

$$
\exists y\left(\bigwedge_{i \in I} x_{i}+k_{i} y \in P \wedge \bigwedge_{j \notin I} x_{j}+k_{j} y \notin P\right),
$$

where $k_{1}, \ldots, k_{n} \in \mathbb{Z}$ and $I \subseteq\{1, \ldots, n\}$. So we have a quantifier-free $L_{ \pm}$-formula $\psi_{\vec{k}, I}(\vec{x})$ such that

$(\star) \forall x_{1} \cdots \forall x_{n}\left(\exists y\left(\bigwedge_{i \in I} x_{i}+k_{i} y \in P \wedge \bigwedge_{j \notin I} x_{j}+k_{j} y \notin P\right) \leftrightarrow \psi_{\vec{k}, I}(\vec{x})\right)$.

holds in $\left(\mathbb{Z},+, 0,1,\left(D_{m,+}^{\mathbb{Z}}\right)_{m \geq 1},\left(D_{m,-}^{\mathbb{Z}}\right)_{m \geq 1}\right)$. As a matter of fact, $\psi_{\vec{k}, I}$ can be chosen to be a formula in the language $L_{P}=\{+,-, 0,1, P\}$.

With this notation at hand, we have the following axiomatization.

Theorem 1.3. Let $\mathcal{M}=\left(M,+,-, 0,1, P^{\mathcal{M}}\right)$ be an $L_{P}$-structure. Then $\mathcal{M}$ is elementarily equivalent to $\left(\mathbb{Z},+,-, 0,1, P_{r}\right)$ if and only if the following hold

(1) $(M,+,-, 0,1) \equiv(\mathbb{Z},+,-, 0,1)$,

(2) for every $k_{1}, \ldots, k_{n} \in \mathbb{Z}$ and $I \subseteq\{1, \ldots, n\}$ the sentence $(\star)$ holds in $\mathcal{M}$,

(3) $k \in P_{r}$ if and only if $k \in P^{\mathcal{M}}$ for every $k \in \mathbb{Z}$.

The technical parts of the proofs are done in an isomorphic structure: Let

$$
\Gamma_{r}:=\left\{\exp \left(\frac{n 2 \pi i}{r}\right) \in \mathbb{C}: n \in \mathbb{Z}\right\}
$$


So $\Gamma_{r}=h(\mathbb{Z})$ where $h$ is the group isomorphism sending $n$ to $\exp \left(\frac{n 2 \pi i}{r}\right)$. Being a subgroup of the unit circle, $\Gamma_{r}$ has an orientation on it; the precise definition is given in the next section. Then the image of $P_{r}$ under $h$ becomes an orientation interval; see Lemma 3.1. This makes it easier to work in $\Gamma_{r}$ and the notations get simpler. For this reason, in Section 2, we recall some facts about the circle and its subgroups.

In Section 3, we introduce Beatty Sequences and prove a few results about them to be used in the model theoretic arguments.

A back-and-forth system constructed in Section 4 is used to prove the theorems mentioned above in the rest of that section and Section 5 .

The paper [9] by Tran-Walsberg has quite a bit of overlap with our work. The authors consider $\mathbb{Z}$ equipped with a 'cyclic ordering'; which in turn is the same as considering an infinite cyclic subgroup of the circle equipped with the orientation. We elaborate on this in Section 5 .

In the last section, we prove a result analogous to the main result of 3]: there are no intermediate structures between $(\mathbb{Z},+)$ and $\left(\mathbb{Z},+, P_{r}\right)$.

Notations and Conventions. The set $\mathbb{N}$ of natural numbers contains 0 and $\mathbb{N}_{+}=\mathbb{N} \backslash\{0\}$. We let the letters $m, n, k, l$ vary in $\mathbb{Z}$, and if $m$ is in $\mathbb{N}\left(\right.$ or $\left.\mathbb{N}_{+}\right)$, we simply write $m \geq 0$ (or $m>0$ ).

For $n>0$, we denote the set $\{1, \ldots, n\}$ as $[n]$.

For a real number $a$, we use the notation $\lfloor a\rfloor$ for the largest integer smaller than or equal to $a$ and $\{a\}$ denotes the difference $a-\lfloor a\rfloor$. (There will not be any occasions where this could be confused with the singleton containing $a$.)

\section{The Circle and Its Subgroups}

In the next section, we work with an infinite cyclic subgroup of the unit circle $\mathbb{S}:=\{\beta \in \mathbb{C}:|\beta|=1\}$. One may study such a group in the generality of oriented abelian groups as defined in [4], however there is no need to do so for our purposes.

Here we recall some generalities about $\mathbb{S}$ and at the end we say a few words about its subgroups. Let

$$
e: \mathbb{R} \rightarrow \mathbb{S}, \quad e(x):=\exp (2 \pi i x) .
$$

This is a surjective group homomorphism with kernel $\mathbb{Z}$.

We equip $\mathbb{S}$ with the counter-clockwise orientation: Given $\alpha, \beta, \gamma \in \mathbb{S}$, the relation $\mathcal{O}(\alpha, \beta, \gamma)$ holds if and only if there are $x, y, z \in \mathbb{R}$ such that $\alpha=e(x), \beta=e(y), \gamma=e(z), x<y<z$, and $z-x<1$. 
If we fix $\alpha \in \mathbb{S}$, then we get a linear ordering $\mathcal{O}_{\alpha}(\cdot, \cdot):=\mathcal{O}(\alpha, \cdot, \cdot)$ on $\mathbb{S} \backslash\{\alpha\}$. For $\alpha=1$, we denote the ordering of $\mathbb{S} \backslash\{1\}$ by the usual ordering sign:

$$
\beta<\gamma \Longleftrightarrow \mathcal{O}(1, \beta, \gamma)
$$

Clearly, if $\alpha<\beta$ and $\beta<\gamma$, then $\mathcal{O}(\alpha, \beta, \gamma)$. So there is no harm in writing

$$
\alpha_{1}<\alpha_{2}<\cdots<\alpha_{n-1}<\alpha_{n}
$$

when $\alpha_{1}<\alpha_{2}, \alpha_{2}<\alpha_{3}, \ldots, \alpha_{n-1}<\alpha_{n}$.

We extend the definition of $<$ to all of $\mathbb{S}$ by setting $1<\alpha$ for $\alpha \neq 1$.

The relation of orientation and the group operation is as follows:

$$
\mathcal{O}(\alpha, \beta, \gamma) \Longleftrightarrow \mathcal{O}(\alpha \delta, \beta \delta, \gamma \delta)
$$

and

$$
\mathcal{O}(\alpha, \beta, \gamma) \Longleftrightarrow \mathcal{O}\left(\gamma^{-1}, \beta,{ }^{-1} \alpha^{-1}\right)
$$

for every $\alpha, \beta, \gamma, \delta \in \mathbb{S}$.

The circle $\mathbb{S}$ has the topology induced by the Euclidean topology on $\mathbb{C}$ and a basis for this topology consists of orientation intervals: Given $\alpha, \beta \in \mathbb{S}$ we define the orientation interval determined by $\alpha$ and $\beta$ to be

$$
(\alpha, \beta):=\{\gamma \in \mathbb{S}: \mathcal{O}(\alpha, \gamma, \beta)\}
$$

We do not assume $\alpha<\beta$ for this definition. So both $(\alpha, \beta)$ and $(\beta, \alpha)$ are orientation intervals and they are disjoint. Such an interval is empty only when $\alpha=\beta$.

We define the length of an orientation interval $I=(\alpha, \beta)$ to be

$$
l(I):=\beta \alpha^{-1} .
$$

So if $\alpha=e(a)$ and $\beta=e(b)$, then the length of $(\alpha, \beta)$ is $e(b-a)$. In particular, the length of an orientation interval is 1 if and only if it is empty.

Below, we use the word interval to mean orientation interval. We also use the notations $[\alpha, \beta),(\alpha, \beta]$, and $[\alpha, \beta]$ with the obvious meanings, and we call them intervals as well.

Proposition 2.1. Let $\alpha_{1}, \ldots, \alpha_{m}, \beta_{1}, \ldots, \beta_{m}$ be distinct elements of $\mathbb{S}$. Then the following conditions are equivalent:

(1) $\bigcap_{i=1}^{m}\left(\alpha_{i}, \beta_{i}\right) \neq \emptyset$.

(2) there is $i_{0} \in[m]$ such that $\alpha_{i_{0}} \in\left(\alpha_{i}, \beta_{i}\right)$ for every $i \neq i_{0}$.

(3) there is $j_{0} \in[m]$ such that $\beta_{j_{0}} \in\left(\alpha_{i}, \beta_{i}\right)$ for every $i \neq j_{0}$.

Moreover, if one of these conditions hold, then

$$
\bigcap_{i=1}^{m}\left(\alpha_{i}, \beta_{i}\right)=\left(\alpha_{i_{0}}, \beta_{j_{0}}\right) \text {. }
$$


Proof. Suppose $\gamma \in\left(\alpha_{i}, \beta_{i}\right)$ for every $i \in[m]$. Then $\mathcal{O}_{\gamma}\left(\beta_{i}, \alpha_{i}\right)$ for each $i \in[m]$. Choose $i_{0}$ such that $\alpha_{i_{0}}$ is maximal among $\alpha_{i}$ with respect to $\mathcal{O}_{\gamma}$. Clearly, $\alpha_{i_{0}} \in\left(\alpha_{i}, \beta_{i}\right)$ for each $i \neq i_{0}$. Similarly, taking $\beta_{j_{0}}$ to be minimum among $\beta_{i}$ with respect to $\mathcal{O}_{\gamma}$ we see that $\beta_{j_{0}} \in\left(\alpha_{i}, \beta_{i}\right)$ for each $i \neq j_{0}$. So the first condition implies the others.

Conversely, assume that $\alpha_{i_{0}} \in\left(\alpha_{i}, \beta_{i}\right)$ for each $i \neq i_{0}$. Then

$$
\left(\alpha_{i_{0}}, \beta_{j_{0}}\right) \subseteq \bigcap_{i=1}^{m}\left(\alpha_{i}, \beta_{i}\right),
$$

where $\beta_{j_{0}}$ is minimum among $\beta_{i}$ with respect to $\mathcal{O}_{\alpha_{i_{0}}}$.

If $\beta_{j_{0}} \in\left(\alpha_{i}, \beta_{i}\right)$ for each $i \neq i_{0}$. Then we take $\alpha_{i_{0}}$ to be maximum among $\alpha_{i}$ with respect to $\mathcal{O}_{\beta j_{0}}$ in order to get

$$
\left(\alpha_{i_{0}}, \beta_{j_{0}}\right) \subseteq \bigcap_{i=1}^{m}\left(\alpha_{i}, \beta_{i}\right) .
$$

The last sentence of the proposition follows from the rest of the proof above.

For $k \in \mathbb{N}_{+}$, let $\zeta_{k}$ denote the primitive $k^{\text {th }}$ root of unity $e\left(\frac{1}{k}\right)$.

Proposition 2.2. Let $\alpha, \beta, \gamma \in \mathbb{S}$ and $k \in \mathbb{N}_{+}$such that $l((\alpha, \gamma))<\zeta_{k}$. Then $\beta^{k} \in\left(\alpha^{k}, \gamma^{k}\right)$ if and only if $\beta \in\left(\zeta_{k}^{s} \alpha, \zeta_{k}^{s} \gamma\right)$ for some $s \in \mathbb{Z}$.

Proof. Note that if $\alpha=\gamma$, then the result is clear; so we assume $\alpha \neq \gamma$. We may also assume that $\alpha<\gamma$; if that is not the case, then multiply $\alpha$ and $\gamma$ by $\zeta_{k}$. So let $0 \leq a<c<1$ be such that $\alpha=e(a)$ and $\gamma=e(c)$. Note that $c-a<\frac{1}{k}$ by assumption. Also let $\beta=e(b)$ for some $0 \leq b<1$.

Suppose $\beta^{k} \in\left(\alpha^{k}, \gamma^{k}\right)$ and take $b^{\prime}$ such that $\beta^{k}=e\left(b^{\prime}\right)$ and $k a<b^{\prime}<$ $k c$. Then $s:=k b-b^{\prime} \in \mathbb{Z}$ and we get

$$
k a+s<k b<k c+s .
$$

Dividing by $k$, we have

$$
a+\frac{s}{k}<b<c+\frac{s}{k}
$$

Applying $e$, we get that $\beta \in\left(\zeta_{k}^{s} \alpha, \zeta_{k}^{s} \gamma\right)$.

Conversely, let $\beta \in\left(\zeta_{k}^{s} \alpha, \zeta_{k}^{s} \gamma\right)$ for some $s \in \mathbb{Z}$. In other words, $\beta \zeta_{k}^{-s} \in$ $(\alpha, \gamma)$. Take $b^{\prime \prime}$ such that $\beta \zeta_{k}^{-s}=e\left(b^{\prime \prime}\right)$ and $a<b^{\prime \prime}<c$. Then $t:=$ $b-b^{\prime \prime}-\frac{s}{k} \in \mathbb{Z}$ and

$$
a+t<b-\frac{s}{k}<c+t
$$

Now multiplying by $k$ and applying $e$ we get $\beta^{k} \in\left(\alpha^{k}, \gamma^{k}\right)$. 
Note that the $s$ in this proposition can be chosen among $0,1, \ldots, k-1$.

Given $\alpha=e(x)$ with $0 \leq x<1$ and $k \in \mathbb{N}_{+}$, we let $\alpha^{1 / k}$ denote $e\left(\frac{x}{k}\right)$; so $\alpha^{1 / k}$ is the $k^{\text {th }}$ root of $\alpha$ with the smallest argument. Note that $1^{1 / k}=1$ and that for $x \in \mathbb{R}$ we have $e(x)^{1 / k}=e\left(\frac{x}{k}\right) \zeta_{k}^{-\lfloor x\rfloor}$. In particular, if $\alpha=e(a)$, then $\left(\alpha^{k}\right)^{1 / k}=\alpha \zeta_{k}^{-\lfloor a k\rfloor}$. However, we always have $\left(\alpha^{1 / k}\right)^{k}=\alpha$. The following observation will be useful.

Lemma 2.3. Let $\alpha, \beta \in \mathbb{S}, k, l \in \mathbb{Z}$. Suppose that $\alpha^{k}=\beta$. Then $\alpha=\beta^{1 / k} \zeta_{k}^{l}$ if and only if $\alpha \in\left(\zeta_{k}^{l}, \zeta_{k}^{l+1}\right)$.

Proof. Clear.

Using this new notation, the previous proposition has the following consequences.

Corollary 2.4. Let $\alpha, \beta, \gamma \in \mathbb{S}$ and $k \in \mathbb{N}_{+}$. Suppose that $\gamma \nless \alpha$. Then $\beta^{k} \in(\alpha, \gamma)$ if and only if there is $s \in\{0,1, \ldots, k-1\}$ such that $\beta \in\left(\zeta_{k}^{s} \alpha^{1 / k}, \zeta_{k}^{s} \gamma^{1 / k}\right)$.

Corollary 2.5. Let $\alpha, \beta, \gamma \in \mathbb{S}$ and $k \in \mathbb{N}_{+}$. Suppose that $\gamma<\alpha$. Then $\beta^{k} \in(\alpha, \gamma)$ if and only if there is $s \in\{0,1, \ldots, k-1\}$ such that $\beta \in\left(\zeta_{k}^{s} \alpha^{1 / k}, \zeta_{k}^{s+1} \gamma^{1 / k}\right)$.

Regularly Dense Groups. Let $\Gamma \leq \mathbb{S}$. Then $\Gamma$ is either finite or dense in $\mathbb{S}$. When it is finite, it consists of $m^{\text {th }}$ roots of unity for some $m>0$. When $\Gamma$ is dense, it is indeed regularly dense in the following sense.

Proposition 2.6. Let $\Gamma \leq \mathbb{S}$ be infinite. Then for any distinct $\alpha, \beta \in \Gamma$ and prime $p$, there is $\gamma \in \Gamma$ such that $\gamma^{p} \in(\alpha, \beta)$.

For the proof of this, we refer the reader to Definition 8.1.7 in [4] and the remark succeeding it. Note that the conclusion of the proposition above is slightly different than the original definition of regularly dense, but it is easy see that they are indeed equivalent. It follows that for any $n>0$ and distinct $\alpha, \beta \in \Gamma$, there is $\gamma \in \Gamma$ such that $\gamma^{n} \in(\alpha, \beta)$.

\section{Beatty Sequences}

Let $r>1$ be an irrational number. The Beatty Sequence generated by $r$ is $\mathcal{B}_{r}=(\lfloor m r\rfloor)_{m>0}$; we put $b_{m}=\lfloor m r\rfloor$. This is an increasing sequence and we let $P_{r}^{+}$denote the set whose elements are the terms of $\mathcal{B}_{r}$.

A related sequence is $\mathcal{S}_{r}=\left(\left\lfloor\frac{n+1}{r}\right\rfloor-\left\lfloor\frac{n}{r}\right\rfloor\right)_{n>0}$; we put $s_{n}=\left\lfloor\frac{n+1}{r}\right\rfloor-\left\lfloor\frac{n}{r}\right\rfloor$. Note that $s_{n} \in\{0,1\}$ for each $n>0$. Actually, it is better to think of $\mathcal{S}_{r}$ as an infinite word in the alphabet $\{0,1\}$. As such, it is called the Characteristic Sturmian Word of Slope $\frac{1}{r}$. It has the property that for every $m$, it has exactly $m+1$ many different subwords of length $m$. 
Both Beatty Sequences and Sturmian Words have rich theories that we do not get into here, and we refer the interested reader to [1]. We only need the following connection between $\mathcal{B}_{r}$ and $\mathcal{S}_{r}$ which is Lemma 9.1.3 of [1], but we include a proof for completeness.

Lemma 3.1. Let $n \in \mathbb{N}_{+}$. Then $n \in P_{r}^{+}$if and only if $s_{n}=1$.

Proof. Let $n \in P_{r}^{+}$. Then $n=\lfloor k r\rfloor$ for some $k \in \mathbb{N}_{+}$. So $k r-1<n<$ $k r$ and after diving by $r$ we have

$$
k-\frac{1}{r}<\frac{n}{r}<k .
$$

Therefore $\left\lfloor\frac{n}{r}\right\rfloor=k-1$ and $\left\lfloor\frac{n+1}{r}\right\rfloor=k$. Thus $s_{n}=\left\lfloor\frac{n+1}{r}\right\rfloor-\left\lfloor\frac{n}{r}\right\rfloor=1$. As all the implications are reversible we get the desired result.

We would like to consider the negative elements as well; so we define

$$
P_{r}=\{\lfloor n r\rfloor: n \in \mathbb{Z} \backslash\{0\}\} .
$$

For $m>0$, we have $-m \in P_{r}$ if and only if $m-1 \in P_{r}^{+}$. So

$$
P_{r}=P_{r}^{+} \cup\left(-P_{r}^{+}-1\right) .
$$

We also extend the definitions of $b_{n}$ and $s_{n}$ to all integers $n$.

Lemma 3.1 is actually correct for all $n \in \mathbb{Z}$ :

$$
n \in P_{r} \Longleftrightarrow s_{n}=1 \text {. }
$$

It is easy to see that $s_{n}=1$ if and only if $\left\{\frac{n}{r}\right\}>1-\frac{1}{r}$. Putting this together with (3.1), for every $n \in \mathbb{Z}$, we get

$$
n \in P_{r} \Longleftrightarrow\left\{\frac{n}{r}\right\}>1-\frac{1}{r} \text {. }
$$

Since $r$ is irrational, the image of $\mathbb{Z} \frac{1}{r}$ under $e$ is not finite, hence it is a dense subgroup of $\mathbb{S}$. Let $\Gamma_{r}$ be that subgroup, and let $h$ denote the map $n \mapsto e\left(\frac{n}{r}\right)$. So we have an isomorphism of abelian groups with a distinguished element:

$$
h:(\mathbb{Z},+,-, 0,1) \simeq\left(\Gamma_{r}, \cdot{ }^{-1}, 1, h(1)\right) .
$$

By (3.2), the image of $P_{r}$ under $h$ is $(h(-1), 1) \cap \Gamma_{r}$. Therefore expanding $\mathbb{Z}$ by $P_{r}$ is the same as expanding $\Gamma_{r}$ by $(h(-1), 1) \cap \Gamma_{r}$.

Using Proposition 2.2 and its corollaries, we give a criterion for certain linear combinations of integers being in $P_{r}$ in terms of intervals in $\mathbb{S}$.

Proposition 3.2. Let $k \in \mathbb{N}_{+}$and $a, c \in \mathbb{Z}$. Then $a+k c \in P_{r}$ if and only if there is $s \in \mathbb{Z}$ such that

$$
h(c) \in\left(h(-a-1)^{1 / k} \zeta_{k}^{s}, h(-a)^{1 / k} \zeta_{k}^{s+s_{a}}\right) .
$$


Proof. First, note that $a \in P_{r}$ if and only if $h(-a)<h(-a-1)$.

By (3.2) we have

$a+k c \in P_{r} \Longleftrightarrow h(a) h(c)^{k} \in(h(-1), 1) \Longleftrightarrow h(c)^{k} \in(h(-a-1), h(-a))$.

Now combining Corollaries 2.4 and 2.5 and using the first sentence of this proof, we get that

$$
h(c)^{k} \in(h(-a-1), h(-a))
$$

if and only if there is $s \in \mathbb{Z}$ with

$$
h(c) \in\left(h(-a-1)^{1 / k} \zeta_{k}^{s}, h(-a)^{1 / k} \zeta_{k}^{s+s_{a}}\right) .
$$

This gives the desired equivalence.

Corollary 3.3. Let $k \in \mathbb{N}_{+}$and $a, c \in \mathbb{Z}$. Then $a+k c \notin P_{r}$ if and only if there is $s \in \mathbb{Z}$ such that

$$
h(c) \in\left[h(-a)^{1 / k} \zeta_{k}^{s+s_{a}}, h(-a-1)^{1 / k} \zeta_{k}^{s+1}\right] .
$$

Proof. Clear from the previous proposition.

Next result will be useful in handling the cases when $k$ is negative.

Lemma 3.4. Let $a, c \in \mathbb{Z}$ and $k<0$. Then $a+k c \in P_{r}$ if and only if $-a-1-k c \in P_{r}$.

Proof. Clear from the fact that $\lfloor-x\rfloor=-\lfloor x\rfloor-1$ for $x \notin \mathbb{Z}$.

Definition 3.5. For $a \in \mathbb{Z}, k \in \mathbb{N}_{+}$, and $s \in\{0,1, \ldots, k-1\}$, let

$$
\begin{aligned}
U_{a, k, s} & :=\left(h(-a-1)^{1 / k} \zeta_{k}^{s}, h(-a)^{1 / k} \zeta_{k}^{s+s_{a}}\right), \\
V_{a, k, s} & :=\left[h(-a)^{1 / k} \zeta_{k}^{s+s_{a}}, h(-a-1)^{1 / k} \zeta_{k}^{s+1}\right] .
\end{aligned}
$$

Also let

$$
U_{a, k}:=\bigcup_{s=0}^{k-1} U_{a, k, s} \quad \text { and } \quad V_{a, k}:=\bigcup_{s=0}^{k-1} V_{a, k, s} .
$$

We extend the definitions to $k=0$ as follows:

$$
U_{a, 0}:=\left\{\begin{array}{ll}
\mathbb{S} & : \text { if } a \in P_{r} \\
\emptyset & : \text { if } a \notin P_{r}
\end{array} \quad \text { and } \quad V_{a, 0}:= \begin{cases}\emptyset & : \text { if } a \in P_{r} \\
\mathbb{S} & : \text { if } a \notin P_{r}\end{cases}\right.
$$

Finally, we let $\tilde{V}_{a, k}$ denote the interior of $V_{a, k}$.

With this notation in hand, Proposition 3.2 and Corollary 3.3 translate as follows: Given $k \in \mathbb{N}$ and $a, c \in \mathbb{Z}$ we have

$$
a+k c \in P_{r} \Longleftrightarrow h(c) \in U_{a, k},
$$

and

$$
a+k c \notin P_{r} \Longleftrightarrow h(c) \in V_{a, k} .
$$


Lemma 3.6. Let $a, b \in \mathbb{Z}$ and $k, l \in \mathbb{N}_{+}$. Suppose $g=\operatorname{gcd}(k, l)$ and write $k=g k^{\prime}$ and $l=g l^{\prime}$. Then the following hold.

(1) Suppose $\zeta_{k^{\prime}}<h(1)$. Then there is $s \in \mathbb{Z}$ such that $h(a)^{1 / k} \zeta_{k}^{s} \in$ $U_{b, l}$.

(2) Suppose $h(1)<\zeta_{k^{\prime}}$. Then there is $s \in \mathbb{Z}$ such that $h(a)^{1 / k} \zeta_{k}^{s} \in$ $U_{b, l}$ if and only if

$$
h\left(l^{\prime} a+k^{\prime} b\right) \in\left(h\left(-k^{\prime}\right), 1\right) .
$$

Proof. Using Corollaries 2.4 and 2.5, $h(a)^{1 / k} \zeta_{k}^{s} \in U_{b, l}$ if and only if

$$
h\left(l^{\prime} a\right)^{1 / k^{\prime}} \zeta_{k^{\prime}}^{\left\lfloor\frac{l a}{r}\right\rfloor-l\left\lfloor\frac{a}{r}\right\rfloor+s l^{\prime}} \in(h(-b-1), h(-b)) .
$$

Since $\operatorname{gcd}\left(k^{\prime}, l^{\prime}\right)=1$, there is $s \in \mathbb{Z}$ with $h(a)^{1 / k} \zeta_{k}^{s} \in U_{b, l}$ if and only if there is $t \in \mathbb{Z}$ with $h\left(l^{\prime} a\right)^{1 / k^{\prime}} \zeta_{k^{\prime}}^{t} \in(h(-b-1), h(-b))$.

If $\zeta_{k^{\prime}}<h(1)$, then there is such a $t$, regardless of what $a, b, l$ are.

If $h(1)<\zeta_{k^{\prime}}$, then we may use Proposition 2.2 to conclude that there is $s \in \mathbb{Z}$ with $h(a)^{1 / k} \zeta_{k}^{s} \in U_{b, l}$ if and only if

$$
h\left(l^{\prime} a\right) \in\left(h\left(-k^{\prime} b-k^{\prime}\right), h\left(-k^{\prime} b\right)\right) .
$$

After simplification, this means $h\left(l^{\prime} a+k^{\prime} b\right) \in\left(h\left(-k^{\prime}\right), 1\right)$.

This proof can be modified to prove the next analogous result.

Lemma 3.7. Let $a, b \in \mathbb{Z}$ and $k, l \in \mathbb{N}_{+}$. Suppose $g=\operatorname{gcd}(k, l)$ and write $k=g k^{\prime}$ and $l=g l^{\prime}$. Then the following hold.

(1) Suppose $\zeta_{k^{\prime}}<h(-1)$. Then there is $s \in \mathbb{Z}$ such that $h(a)^{1 / k} \zeta_{k}^{s} \in$ $V_{b, l}$.

(2) Suppose $h(-1)<\zeta_{k^{\prime}}$. Then there is $s \in \mathbb{Z}$ such that $h(a)^{1 / k} \zeta_{k}^{s} \in$ $V_{b, l}$ if and only if

$$
h\left(l^{\prime} a+k^{\prime} b^{\prime}\right) \in\left[1, h\left(-k^{\prime}\right)\right]
$$

Let $\vec{a}=\left(a_{1}, \ldots, a_{n}\right) \in \mathbb{Z}^{n}, \vec{k}=\left(k_{1}, \ldots, k_{n}\right) \in \mathbb{N}^{n}, I \subseteq[n]$ and $J \subseteq[n] \backslash I$ be given. We define

$$
V_{\vec{a}, \vec{k}, J}=\bigcap_{j \in J}\left(V_{a_{j}, k_{j}} \backslash \tilde{V}_{a_{j}, k_{j}}\right) \cap \bigcap_{j \in[n] \backslash(I \cup J)} \tilde{V}_{a_{j}, k_{j}} .
$$

Note that $V_{a_{j}, k_{j}} \backslash \tilde{V}_{a_{j}, k_{j}}$ has $2 k_{j}$ many points. Therefore $V_{\vec{a}, \vec{k}, J}$ is finite for $J \neq \emptyset$ and $V_{\vec{a}, \vec{k}, \emptyset}$ is an open subset of $\mathbb{S}$.

We record the following without proof.

Lemma 3.8. Let $\vec{a}=\left(a_{1}, \ldots, a_{n}\right) \in \mathbb{Z}^{n}, \vec{k}=\left(k_{1}, \ldots, k_{n}\right) \in \mathbb{N}_{+}^{n}$, and $\emptyset \neq J \subseteq[n]$. Then $h(c) \in V_{\vec{a}, \vec{k}, J} \cap \Gamma_{r}$ if and only if there is a subset $J^{\prime}$ of $J$ such that $c=\frac{-a_{j}}{k_{j}}$ for every $j \in J^{\prime}, c=\frac{-a_{j}-1}{k_{j}}$ for every $j \in J \backslash J^{\prime}$, and $h(c) \in \bigcap_{j \in[n] \backslash J} \tilde{V}_{a_{j}, k_{j}}$. 
Definition 3.9. Let $\vec{k}=\left(k_{1}, \ldots, k_{n}\right) \in \mathbb{Z}^{n}$ and $I \subseteq[n]$. We say that $\left(a_{1}, \ldots, a_{n}\right) \in \mathbb{Z}^{n}$ realize $(\vec{k}, I)$-pattern if there is $c \in \mathbb{Z}$ such that

$$
a_{i}+k_{i} c \in P_{r} \Longleftrightarrow i \in I \text {. }
$$

For $\vec{k}=\left(k_{1}, \ldots, k_{n}\right) \in \mathbb{Z}^{n}$, let $|\vec{k}|=\left(\left|k_{1}\right|, \ldots,\left|k_{n}\right|\right)$. Then using Lemma 3.4, $\left(a_{1}, \ldots, a_{n}\right) \in \mathbb{Z}^{n}$ realizes the $(\vec{k}, I)$-pattern if $\left(a_{1}^{\prime}, \ldots, a_{n}^{\prime}\right)$ realizes the $(|\vec{k}|, I)$-pattern where $a_{i}^{\prime}=a_{i}$ for $k_{i} \geq 0$ and $a_{i}^{\prime}=-a_{i}-1$ for $k_{i}<0$. Therefore, we may focus on the case that $\vec{k} \in \mathbb{N}^{n}$.

By (3.3) and (3.4), if $\vec{k} \in \mathbb{N}^{n}$, then $\left(a_{1}, \ldots, a_{n}\right) \in \mathbb{Z}^{n}$ realize $(\vec{k}, I)$ pattern if and only if

$$
\bigcap_{i \in I} U_{a_{i}, k_{i}} \cap \bigcap_{j \notin I} V_{a_{j}, k_{j}} \cap \Gamma_{r} \neq \emptyset
$$

We may decompose the intersection above as

$$
\left(\bigcap_{i \in I} U_{a_{i}, k_{i}} \cap \bigcap_{j \notin I} \tilde{V}_{a_{j}, k_{j}} \cap \Gamma_{r}\right) \cup\left(\bigcap_{i \in I} U_{a_{i}, k_{i}} \cap \bigcup_{\emptyset \neq J \subseteq[n] \backslash I} V_{\vec{a}, \vec{k}, J} \cap \Gamma_{r}\right) .
$$

Using Lemma 3.8, the finite component is under control.

Let's focus on $\bigcap_{i \in I} U_{a_{i}, k_{i}} \cap V_{\vec{a}, \vec{k}, \emptyset} \cap \Gamma_{r}$. Since $\Gamma_{r}$ is dense in $\mathbb{S}$, this set is nonempty if and only if the open set

$$
\bigcap_{i \in I} U_{a_{i}, k_{i}} \cap \bigcap_{j \notin I} \tilde{V}_{a_{j}, k_{j}}
$$

is nonempty.

Using Proposition 2.1, it is easy to see that this intersection is nonempty if and only if one of the following holds:

(1) there are $i_{0} \in I$ and $s \in \mathbb{Z}$ such that

$$
h\left(-a_{i_{0}}-1\right)^{1 / k_{i_{0}}} \zeta_{k_{i_{0}}}^{s} \in \bigcap_{i \in I, i \neq i_{0}} U_{a_{i}, k_{i}} \cap \bigcap_{j \notin I} \tilde{V}_{a_{j}, k_{j}}
$$

(2) there are $j_{0} \in[n] \backslash I$ and $s \in \mathbb{Z}$ such that

$$
h\left(-a_{j_{0}}\right)^{1 / k_{j_{0}}} \zeta_{k_{j_{0}}}^{s} \in \bigcap_{i \in I} U_{a_{i}, k_{i}} \cap \bigcap_{j \notin I, j \neq j_{0}} \tilde{V}_{a_{j}, k_{j}} .
$$

In order to summarize these observations, we make the following definitions: let $k, l \in \mathbb{N}$ with $g=\operatorname{gcd}(k, l)$ and $k^{\prime}:=k / g, l^{\prime}:=l / g$

$$
A_{k, l}:= \begin{cases}\mathbb{Z} \times \mathbb{Z} & : \text { if } \zeta_{k^{\prime}}<h(1) \\ \left\{(a, b) \in \mathbb{Z} \times \mathbb{Z}: h\left(l^{\prime} a+k^{\prime} b\right) \in\left(h\left(-k^{\prime}\right), 1\right)\right\} & : \text { if } h(1)<\zeta_{k^{\prime}}\end{cases}
$$


$B_{k, l}:= \begin{cases}\mathbb{Z} \times \mathbb{Z} & : \text { if } \zeta_{k^{\prime}}<h(-1) \\ \left\{(a, b) \in \mathbb{Z} \times \mathbb{Z}: h\left(l^{\prime} a+k^{\prime} b\right) \in\left(1, h\left(-k^{\prime}\right)\right)\right\} & : \text { if } h(-1)<\zeta_{k^{\prime}}\end{cases}$

Combining Lemmas 3.6 and 3.7 with the observations above, we obtain the following.

Proposition 3.10. Let $\vec{k}=\left(k_{1}, \ldots, k_{n}\right) \in \mathbb{N}^{n}$ and $I \subseteq[n]$. Then $\vec{a}=\left(a_{1}, \ldots, a_{n}\right) \in \mathbb{Z}^{n}$ realizes the $(\vec{k}, I)$-pattern if and only if one of the following conditions holds:

(1) $\bigcap_{i \in I} U_{a_{i}, k_{i}} \cap \bigcup_{\emptyset \neq J \subseteq[n] \backslash I} V_{\vec{a}, \vec{k}, J} \cap \Gamma_{r} \neq \emptyset$.

(2) there is $i_{0} \in I$ with $\left(-a_{i_{0}}-1, a_{i}\right) \in A_{k_{i_{0}}, k_{i}}$ for every $i \in I \backslash\left\{i_{0}\right\}$ and $\left(-a_{i_{0}}-1, a_{j}\right) \in B_{k_{i_{0}}, k_{j}}$ for every $j \in[n] \backslash I$.

(3) there is $j_{0} \in I$ with $\left(-a_{j_{0}}, a_{i}\right) \in A_{k_{j_{0}}, k_{i}}$ for every $i \in I$ and $\left(-a_{j_{0}}, a_{j}\right) \in B_{k_{j_{0}}, k_{j}}$ for every $j \in[n] \backslash\left(I \cup\left\{j_{0}\right\}\right)$.

The final result of this section expresses the interval $(h(k), 1)$ in terms of $P_{r}$ when $h(1)<\zeta_{k}$.

Lemma 3.11. Let $k>0$ be such that $h(1)<\zeta_{k}$. Then $\alpha \in(1, h(k))$ if and only if

$$
\alpha^{-1} \in \bigcup_{i=0}^{k-1} h(-i) P_{r} \cup\{h(-1), h(-2), \ldots, h(-(k-1))\} .
$$

(Here $h(i) P_{r}$ is short for the interval $(h(-i-1), h(-i))$.)

Proof. The assumption $h(1)<\zeta_{k}$ gives $h(i-1)<h(i)$ for every $i \in[k]$. So we have the decomposition

$$
\begin{aligned}
(1, h(k)) & =(1, h(1)] \cup(h(1), h(2)] \cup \cdots \cup(h(k-1), h(k)) \\
& =(1, h(1)) \cup \cdots \cup(h(k-1), h(k)) \cup\{h(1), \ldots, h(k-1)\} \\
& =\bigcup_{i=0}^{k-1} h(i)(1, h(1)) \cup\{h(1), \ldots, h(k-1)\}
\end{aligned}
$$

This finishes the proof, since $\beta \in(1, h(1))$ if and only if $\beta^{-1} \in P_{r}$.

\section{Expanding the Group of Integers}

We would like to consider the model theoretic structure obtained by expanding the abelian group of integers by the subset $P_{r}$. We have seen above that $\mathbb{Z}$ is isomorphic as an abelian group with a subgroup $\Gamma_{r}$ of $\mathbb{S}$ that happens to be dense in $\mathbb{S}$. The work in the previous section was mostly done in $\Gamma_{r}$, but it is straightforward to pull those results back to $\mathbb{Z}$ via the map $h$.

Let $L:=\{+,-, 0, c\}$ be the language of abelian groups with a distinguished element $c$. Let $T$ be the theory of the $L$-structure $(\mathbb{Z},+,-, 0,1)$. 
We extend $L$ to $L_{P}:=L \cup\{P\}$ where $P$ is a unary relation symbol. Our main objective is to study the $L_{P}$-structure

$$
\mathfrak{Z}:=\left(\mathbb{Z},+,-, 0,1, P_{r}\right) \text {. }
$$

For $\vec{k}=\left(k_{1}, \ldots, k_{n}\right) \in \mathbb{Z}^{n}$ and $I \subseteq[n]$, we define $\phi_{\vec{k}, I}\left(x_{1}, \ldots, x_{n}\right)$ to be the following $L_{P}$-formula:

$$
\exists y\left(\bigwedge_{i \in I} x_{i}+k_{i} y \in P \wedge \bigwedge_{j \notin I} x_{j}+k_{j} y \notin P\right) .
$$

Therefore for $\left(a_{1}, \ldots, a_{n}\right) \in \mathbb{Z}^{n}$, we have $\mathfrak{Z} \models \phi_{\vec{k}, I}\left(a_{1}, \ldots, a_{n}\right)$ if and only if $\left(a_{1}, \ldots, a_{n}\right)$ realizes the $(\vec{k}, I)$-pattern. Then using Proposition 3.10 and Lemma 3.11, there is a quantifier-free $L_{P}$-formula $\psi_{\vec{k}, I}\left(x_{1}, \ldots, x_{n}\right)$ such that

$$
\mathfrak{Z} \models \forall x_{1} \cdots \forall x_{n}\left(\phi_{\vec{k}, I}\left(x_{1}, \ldots, x_{n}\right) \leftrightarrow \psi_{\vec{k}, I}\left(x_{1}, \ldots, x_{n}\right)\right)
$$

Let $T_{r}$ be the $L_{P}$-theory extending $T$ by the condition above; namely for every $\vec{k}=\left(k_{1}, \ldots, k_{n}\right) \in \mathbb{Z}^{n}$ and $I \subseteq[n]$, we add the following sentence as an axiom:

$$
\forall x_{1} \cdots \forall x_{n}\left(\phi_{\vec{k}, I}\left(x_{1}, \ldots, x_{n}\right) \leftrightarrow \psi_{\vec{k}, I}\left(x_{1}, \ldots, x_{n}\right)\right)
$$

We shall construct a back-and-forth system between certain substructures of models of $T_{r}$.

Let $\mathcal{M}$ and $\mathcal{N}$ be $\aleph_{0}$-saturated models of $T_{r}$. Let $S_{\mathcal{M}}$ be the collection of

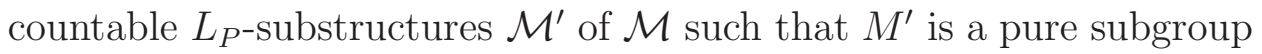
of $M$. We define $S_{\mathcal{N}}$ in a similar way.

Note that the group $\mathbb{Z}$ has a copy in each member of $S_{\mathcal{M}}$ and $S_{\mathcal{N}}$ as the subgroup generated by the constant $c$. However, those copies may not be isomorphic as $L_{P}$-substructures of $\mathcal{M}$ and $\mathcal{N}$.

Let $\mathfrak{B}(\mathcal{M}, \mathcal{N})$ be the collection of $L_{P^{-}}$isomorphisms $f: \mathcal{M}^{\prime} \rightarrow \mathcal{N}^{\prime}$, where $\mathcal{M}^{\prime} \in S_{\mathcal{M}}$ and $\mathcal{N}^{\prime} \in S_{\mathcal{N}}$.

Proposition 4.1. For $\aleph_{0}$-saturated models $\mathcal{M}$ and $\mathcal{N}$ of $T_{r}$, the collection $\mathfrak{B}(\mathcal{M}, \mathcal{N})$ is a back-and-forth system.

Proof. Let $f: \mathcal{M}^{\prime} \rightarrow \mathcal{N}^{\prime}$ be in $\mathfrak{B}(\mathcal{M}, \mathcal{N})$ and $\alpha \in M \backslash M^{\prime}$. By symmetry, it suffices to extend $f$ to an element of $\mathfrak{B}(\mathcal{M}, \mathcal{N})$ that contains $\alpha$ in its domain.

Let $M^{\prime \prime}$ be the pure subgroup of $M$ generated by $M^{\prime}$ and $\alpha$; namely:

$$
M^{\prime \prime}=\left\langle M^{\prime} \cup\{\alpha\}\right\rangle_{M}:=\left\{\gamma \in M: m \gamma \in M^{\prime} \oplus \mathbb{Z} \alpha \text { for some } m>0\right\} .
$$

Also let $\mathcal{M}^{\prime \prime}$ be the $L_{P}$-substructure of $\mathcal{M}$ with the underlying set $M^{\prime \prime}$. We would like to extend $f$ to $\mathcal{M}^{\prime \prime}$. That amounts to finding $\beta \in N$ with the following property: 
(*) For every $a \in M^{\prime}, k \in \mathbb{Z}, n>0$, and $\gamma \in M$ if $a+k \alpha=n \gamma$, then there is $\delta \in N$ such that $f(a)+k \beta=n \delta$, and

$$
\gamma \in P \Longleftrightarrow \delta \in P \text {. }
$$

This condition without the last part just means that $\left\langle M^{\prime} \cup\{\alpha\}\right\rangle_{M}$ and $\left\langle N^{\prime} \cup\{\beta\}\right\rangle_{N}$ are isomorphic as groups. Since the reducts of $\mathcal{M}$ and $\mathcal{N}$ to $L$ are models of $T$, there is certainly such an element $\beta$ in $N$. So the point is to find $\beta$ in a way that that isomorphism of groups is indeed an $L_{P}$-isomorphism.

By saturation, it suffices to find $\beta \in N$ satisfying a given finite fragment of $(*)$. So let $a_{1}, \ldots, a_{m} \in M^{\prime}, k_{1}, \ldots, k_{m} \in \mathbb{Z}, n_{1}, \ldots, n_{m} \in \mathbb{N}_{+}$, and $\gamma_{1}, \ldots, \gamma_{m} \in M$ be such that $a_{i}+k_{i} \alpha=n_{i} \gamma_{i}$ for each $i$. Then we need to find $\beta, \delta_{1}, \ldots, \delta_{m} \in N$ such that $f\left(a_{i}\right)+k_{i} \beta=n_{i} \delta_{i}$ and $\delta_{i} \in P$ if and only if $\gamma_{i} \in P$ for every $i$.

Let $\nu=\operatorname{lcm}\left(n_{1}, \ldots, n_{m}\right)$, and let $d \in\{0, \ldots, \nu-1\}$ and $\alpha^{\prime} \in M$ be such that $\alpha=d+\nu \alpha^{\prime}$. Then $\gamma_{i}=a_{i}^{\prime}+k_{i}^{\prime} \alpha^{\prime}$, where $n_{i} a_{i}^{\prime}=a_{i}+k_{i} d$ and $k_{i}^{\prime}=\frac{k \nu}{n_{i}}$. Since $M^{\prime}$ is pure in $M$, it contains $a_{i}^{\prime}$. Therefore it suffices to find $\beta^{\prime} \in N$ such that for every $i$ :

$$
a_{i}^{\prime}+k_{i}^{\prime} \alpha^{\prime} \in P \Longleftrightarrow f\left(a_{i}^{\prime}\right)+k_{i}^{\prime} \beta^{\prime} \in P
$$

Taking $\vec{k}=\left(k_{1}^{\prime}, \ldots, k_{n}^{\prime}\right)$ and $I=\left\{i: a_{i}^{\prime}+k_{i}^{\prime} \alpha^{\prime} \in P\right\}$, we have

$$
\mathcal{M} \models \phi_{\vec{k}, I}\left(a_{1}^{\prime}, \ldots, a_{n}^{\prime}\right) .
$$

Hence

$$
\mathcal{M} \models \psi_{\vec{k}, I}\left(a_{1}^{\prime}, \ldots, a_{n}^{\prime}\right) \text { and } \mathcal{M}^{\prime} \models \psi_{\vec{k}, I}\left(a_{1}^{\prime}, \ldots, a_{n}^{\prime}\right) .
$$

Thus

$$
\mathcal{N}^{\prime} \models \psi_{\vec{k}, I}\left(f\left(a_{1}^{\prime}\right), \ldots, f\left(a_{n}^{\prime}\right)\right) \text { and } \mathcal{N} \models \psi_{\vec{k}, I}\left(f\left(a_{1}^{\prime}\right), \ldots, f\left(a_{n}^{\prime}\right)\right) .
$$

As a result $\mathcal{N} \models \phi_{\vec{k}, I}\left(f\left(a_{1}^{\prime}\right), \ldots, f\left(a_{n}^{\prime}\right)\right)$ and hence there is $\beta^{\prime} \in N$ with the desired property:

$$
f\left(a_{i}^{\prime}\right)+k_{i}^{\prime} \beta^{\prime} \in P \Longleftrightarrow i \in I \Longleftrightarrow a_{i}^{\prime}+k_{i}^{\prime} \alpha^{\prime} \in P .
$$

4.1. Quantifier Elimination and Axiomatization. The theory $T_{r}$ does not have quantifier elimination for the obvious reason that for any $n>1$, the definable subgroup consisting of elements divisible by $n$ is not quantifier-free definable. However, we still do not get quantifier elimination after adding predicate symbols to represent those subgroups, because we also need to know whether the element obtained by dividing by $n$ is in $P$ or not. So for every $n \geq 1$ we add two new unary predicate symbols $D_{n,+}$ and $D_{n,-}$ to the language $L_{P}$ to obtain 
$L_{ \pm}$and let $T_{ \pm}$be the definitional extension of $T_{r}$ to an $L_{ \pm}$-theory by adding the following for each $n \geq 1$ :

$$
\begin{aligned}
& \forall x\left(D_{n,+}(x) \leftrightarrow \exists y(x=n y \wedge y \in P)\right) \\
& \forall x\left(D_{n,-}(x) \leftrightarrow \exists y(x=n y \wedge y \notin P)\right)
\end{aligned}
$$

Therefore, every model $\mathcal{M}$ of $T_{r}$ expands to a model of $T_{ \pm}$; we still denote this extension by $\mathcal{M}$. Note that for a model $\mathcal{M}$ of $T_{ \pm}$, we have $D_{n,+}(\mathcal{M}) \cup D_{n,-}(\mathcal{M})=n M$ for every $n \geq 1$ and $D_{1,+}(\mathcal{M})=P(\mathcal{M})$.

Now we are ready to prove Theorem 1.2 in a stronger form.

Theorem 4.2. The theory $T_{ \pm}$has quantifier elimination.

Proof. It suffices to prove the following:

$(\dagger)$ Let $\mathcal{M}$ and $\mathcal{N}$ be models of $T_{ \pm}$and $\mathcal{A}$ a common finitely generated $L_{ \pm}$-substructure of $\mathcal{M}$ and $\mathcal{N}$. Then $\mathcal{M} \equiv_{A} \mathcal{N}$. (This means that

$\mathcal{M}$ and $\mathcal{N}$ are elementarily equivalent as $L_{ \pm}(A)$-structures )

(For why this is enough, see, for instance, Proposition 18.2 of [5].)

We may assume that $\mathcal{M}$ and $\mathcal{N}$ are $\aleph_{0}$-saturated. Let

$$
M^{\prime}=\langle A\rangle_{M} \text { and } N^{\prime}=\langle A\rangle_{N} .
$$

Clearly, $M^{\prime}$ and $N^{\prime}$ are isomorphic as abelian groups via a map extending the identity map on $A$. If $n \alpha=a$ for some $a \in A$ and $\alpha \in M$, then there is $\beta \in N$ with $n \beta=a$. Then the isomorphism sends $\alpha$ to $\beta$. But we also have $\alpha \in P(\mathcal{M})$ if and only if $\beta \in P(\mathcal{N})$, since either both $\mathcal{M}$ and $\mathcal{N}$ satisfy $D_{n,+}(a)$ or they both satisfy $D_{n,-}(a)$. Therefore $M^{\prime}$ and $N^{\prime}$ are underlying sets of $L_{ \pm}$-substructures $\mathcal{M}^{\prime}$ and $\mathcal{N}^{\prime}$ of $\mathcal{M}$ and $\mathcal{N}$ respectively, and they are isomorphic. Since $M^{\prime}$ and $N^{\prime}$ are countable and pure in $M$ and $N$, that isomorphism is in $\mathfrak{B}(\mathcal{M}, \mathcal{N})$. It follows that $\mathcal{M} \equiv_{M^{\prime}} \mathcal{N}$ and in particular $\mathcal{M} \equiv_{A} \mathcal{N}$.

Given $\aleph_{0}$-saturated models $\mathcal{M}$ and $\mathcal{N}$ of $T_{r}$, we may still have that $\mathfrak{B}(\mathcal{M}, \mathcal{N})=\emptyset$. So in order to get completeness we extend $T_{ \pm}$to $T_{r}^{*}$ by adding $\mathbb{Z}$-axioms: Given $k \in \mathbb{N}_{+}$if $k \in P_{r}$, then we add the axiom $k \in P$, otherwise we add the axiom $k \notin P$. (Recall that 1 is in the language, so $k=1+\cdots+1$.) Clearly, $T_{r}^{*}$ still has quantifier elimination.

With this extension, we get Theorem 1.3 .

Theorem 4.3. The theory $T_{r}^{*}$ is complete.

Proof. Clearly, $\mathfrak{Z}$ is an algebraically prime model of $T_{r}^{*}$. Since $T_{r}^{*}$ has quantifier elimination, we get that $T_{r}^{*}$ is complete.

Question. According to this theorem each $T_{r}^{*}$ is a completion of $T_{r}$. Is it correct that each completion of $T_{r}$ is given as the theory of an expansion of the group of integers by a Beatty Sequence? 


\section{Definable Sets}

When dealing with the definable sets, we constantly switch between $\mathfrak{Z}$ and the isomorphic structure

$$
\mathfrak{G}:=\left(\Gamma_{r}, \cdot{ }^{-1}, 1, h(1), P_{r}\right),
$$

where $P_{r}$ denotes the interval $(h(-1), 1) \cap \Gamma_{r}$ of $\Gamma_{r}$.

For a subset $X$ of $\Gamma_{r}^{n}$ and $m>0$ we let

$$
\begin{aligned}
& X^{(m)}:=\left\{\left(x_{1}^{m}, \ldots, x_{n}^{m}\right): \vec{x} \in X\right\}, \text { and } \\
& X^{1 / m}:=\left\{\vec{y} \in \Gamma_{r}^{n}:\left(y_{1}^{m}, \ldots, y_{n}^{m}\right) \in X\right\} .
\end{aligned}
$$

For a subset $A$ of $\mathbb{S}$, we define $[A]$ to be the subgroup of $\mathbb{S}$ generated by $A$ and

$$
\langle A\rangle:=\left\{\alpha \in \mathbb{S}: \alpha^{m} \in[A] \text { for some } m>0\right\} .
$$

We collect some easy facts about these notions.

Lemma 5.1. Let $X, Y \subseteq \Gamma_{r}^{n}$ and $m>0$.

(1) $D_{m,+}^{1 / m}=P_{r}$ and $P_{r}^{(m)}=D_{m,+}$.

(2) $\left(X^{(m)}\right)^{1 / m}=X$ and $\left(X^{1 / m}\right)^{(m)}=X \cap\left(\Gamma_{r}^{n}\right)^{(m)}$

(3) $(X \cap Y)^{1 / m}=X^{1 / m} \cap Y^{1 / m},(X \cup Y)^{1 / m}=X^{1 / m} \cup Y^{1 / m}$, and $\left(\Gamma_{r}^{n} \backslash X\right)^{1 / m}=\Gamma_{r}^{n} \backslash X^{1 / m}$

(4) For $\vec{a} \in(\{0, \ldots, m-1\})^{n}$, let $X_{\vec{a}}=h(\vec{a}) X \cap\left(\Gamma_{r}^{n}\right)^{(m)}$. Then

$$
X=\bigcup_{\vec{a}} h(-\vec{a}) X_{\vec{a}} .
$$

Before analyzing definable sets in detail, we would like to clarify the connection with the paper [9].

Lemma 5.2. Let $m>0$ and let $X=(\alpha, \beta) \cap \Gamma_{r}^{(m)}$. Then any set $\left(\alpha \gamma_{1}, \beta \gamma_{2}\right) \cap \Gamma_{r}^{(m)}$ with $\gamma_{1}, \gamma_{2} \in \Gamma_{r}^{(m)}$ is definable in $\left(\Gamma_{r}, \cdot, X\right)$.

Proof. It suffices to show that $Y=(\alpha, \beta \gamma) \cap \Gamma_{r}^{(m)}$ is definable in $\left(\Gamma_{r}, \cdot, X\right)$, where $\gamma=\gamma_{2} \gamma_{1}^{-1}$.

Let $\lambda=\beta \alpha^{-1}$ and take $\delta \in\left(\lambda^{1 / 2}, \lambda\right) \cap \Gamma_{r}^{(m)}$. Suppose that $n$ is the largest natural number such that $\gamma \delta^{-n}<\lambda$.

Then $Y$ is the following union:

$$
Y=X \cup \delta X \cup \cdots \cup \delta^{n} X \cup\left(\left(\alpha \delta^{n+1}, \beta \gamma\right) \cap \Gamma_{r}^{(m)}\right),
$$

Note that

$$
\left(\alpha \delta^{n+1}, \beta \gamma\right) \cap \Gamma_{r}^{(m)}=\delta^{n+1}\left(X \cap \gamma\left(\delta^{n+1}\right)^{-1} X\right) .
$$

So $Y$ is definable in $\left(\Gamma_{r}, \cdot, X\right)$.

Letting $X=P_{r}$ in this lemma, we have the following consequence. 
Corollary 5.3. Any interval of $\Gamma_{r}$ is definable in $\mathfrak{G}$.

Therefore, the structures $\mathfrak{G}$ and $\left(\Gamma_{r}, \cdot, \mathcal{O}\right)$ are interdefinable, where $\mathcal{O}$ is the restriction of the orientation of $\mathbb{S}$ to $\Gamma_{r}$. In [9], the authors study the latter structure; or rather the pull-back of it under $h$. Even though some of our results are slightly finer than theirs, they also work out some stability theoretic properties of this structure. Most notably, they show that it is dp-minimal.

We appeal to the topology on $\Gamma_{r}^{n}$ induced from $\mathbb{S}^{n}$ in order to study definable sets. An open basis for that topology on $\Gamma_{r}$ consists of sets of the form $I \cap \Gamma_{r}$ where $I$ is an open interval of $\mathbb{S}$; below we refer to these sets as convex sets. From now on we use the word interval to mean open interval of $\Gamma_{r}$; so an interval is a convex set whose end points are in $\Gamma_{r}$.

The quantifier elimination result in the previous section gives a very simple characterization of definable sets in a model $\mathcal{M}$ of $T_{r}^{*}$ : They are Boolean combinations of sets defined by formulas of the form

$$
a+\vec{k} \vec{x}=0, D_{m,+}(a+\vec{k} \vec{x}), \text { and } D_{m,-}(a+\vec{k} \vec{x})
$$

where $\vec{x}=\left(x_{1}, \ldots, x_{n}\right)$ is a tuple of variables, $a \in M$ and $\vec{k} \in \mathbb{Z}^{n}$.

It is simpler in the sense that the negations of formulas $D_{m,+}(a+\vec{k} \vec{x})$ and $D_{m,-}(a+\vec{k} \vec{x})$ define sets that are finite unions of sets defined by the same kind of formulas. Also we would like to consider the formula

$$
D_{m,-}(a+\vec{k} \vec{x}) \wedge a+\vec{k} \vec{x} \neq 0 \wedge a+\vec{k} \vec{x} \neq-m
$$

in the place of $D_{m,-}(a+\vec{k} \vec{x})$.

So any definable set in $\mathfrak{G}$ is of the form

$$
\bigcup_{i=1}^{s} \bigcap_{j=1}^{t_{i}} X_{i j},
$$

where each $X_{i j}$ is one of the following forms

$$
\begin{gathered}
\left\{\vec{x} \in \Gamma_{r}^{n}: \vec{x}^{\vec{k}} h(a)=1\right\} \\
\left\{\vec{x} \in \Gamma_{r}^{n}: \vec{x}^{\vec{k}} h(a) \neq 1\right\} \\
\left\{\vec{x} \in \Gamma_{r}^{n}: \vec{x}^{\vec{k}} h(a) \in P_{r}^{(m)}\right\} \\
\left\{\vec{x} \in \Gamma_{r}^{n}: \vec{x}^{k} h(a) \in Q_{r}^{(m)}\right\}
\end{gathered}
$$


where $\vec{k}=\left(k_{1}, \ldots, k_{n}\right) \in \mathbb{Z}^{n} \backslash\{\overrightarrow{0}\}, a \in \mathbb{Z}, m>0, \vec{x}^{k}:=x_{1}^{k_{1}} x_{2}^{k_{2}} \cdots x_{n}^{k_{n}}$, and $Q_{r}=(1, h(-1)) \cap \Gamma_{r}$.

Note that a set of the form (A) is nonempty if and only if $\kappa \mid a$ where $\kappa$ is the greatest common divisor of the integers $k_{i}$. We refer to a finite intersection of sets of the form (A) as an affine subset of $\Gamma_{r}^{n}$ provided that it is nonempty. Affine subsets of $\Gamma_{r}$ are singletons, and if $Y$ is an affine subset of $\Gamma_{r}^{n}$ with $n>1$, then there is a projection $\pi: \Gamma_{r}^{n} \rightarrow \Gamma_{r}^{d}$ with $d<n$ such that $\left.\pi\right|_{Y}$ is injective. Also for an affine subset $Y$, $\vec{b} \in \mathbb{Z}^{n}$, and $N>0$, the set $(h(\vec{b}) Y)^{1 / N}$ is either empty or an affine subset.

Suppose that $Y$ is a set of the form (C) or (D), $\vec{b} \in \mathbb{Z}^{n}$, and let $N \in \mathbb{N}_{+}$ be a multiple of $m$. Then the set $(h(\vec{b}) Y)^{1 / N}$ is nonempty if and only if $m \mid a-\vec{k} \vec{b}$, and in that case

$$
(h(\vec{b}) Y)^{1 / N}=\left\{\vec{y}:\left(\vec{y}^{\vec{k}}\right)^{N / m} \in h\left(\frac{\vec{k} \vec{b}-a}{m}\right) I\right\},
$$

where $I$ is one of $P_{r}$ or $Q_{r}$. As a result, it is an open subset of $\Gamma_{r}^{n}$.

Putting these together we have the following result.

Proposition 5.4. Let $X$ be definable in $\mathfrak{G}$. Then there is $N \in \mathbb{N}_{+}$ such that for every $\vec{b} \in \mathbb{Z}^{n}$ the set $(h(\vec{b}) X)^{1 / N}$ is a union of an open set and finitely many subsets of affine sets.

Proof. Write $X$ as in (*), and let $N$ be the lowest common multiple of the integers $m$ appearing in the formulas defining the sets $X_{i j}$. Given $i \in\{1, \ldots, s\}$, if one of the sets $X_{i j}$ is of the form (A), then the set

$$
\left(h(\vec{b}) \bigcap_{j} X_{i j}\right)^{1 / N}=\bigcap_{j}\left(h(\vec{b}) X_{i j}\right)^{1 / N}
$$

is contained in an affine set. Otherwise this set is open as noted above.

Now we focus on unary definable sets with the aim of proving Theorem 1.1. Combined with Proposition 2.2. Proposition 5.4 gives the following for unary subsets of $\Gamma_{r}$.

Corollary 5.5. Suppose that $X \subseteq \Gamma_{r}$ is definable in $\mathfrak{G}$. Then there is $N \in \mathbb{N}_{+}$such that for every $n \in\{0,1, \ldots, N-1\}$, the set $(h(n) X)^{1 / N}$ is a finite union of convex sets and singletons. Moreover, the end points of the convex sets are in $\left\langle\Gamma_{r}\right\rangle$.

Proof. Affine subsets of $\Gamma_{r}$ are singletons and the open set appearing in $(h(n) X)^{1 / N}$ is a certain positive Boolean combination of sets of the form:

$$
\left\{y \in \Gamma_{r}: y^{k N} \neq h(-n)\right\} \text {. }
$$


and

where $I$ is one of $P_{r}$ or $Q_{r}$.

$$
\left\{y \in \Gamma_{r}: y^{k N / m} \in h\left(\frac{k b-n}{m}\right) I\right\}
$$

Using Proposition 2.2, if such a combination is not empty, then it is a finite union of convex sets whose end points are in $\left\langle\Gamma_{r}\right\rangle$.

Definition 5.6. Let $X \subseteq \mathbb{Z}$. We say that $X$ has the uniform gaps property if there is $M \in \mathbb{N}_{+}$such that

$$
X \cap\{x+1, \ldots, x+M\} \neq \emptyset
$$

for every $x \in X$.

Clearly, $\emptyset$ has the uniform gaps property and it is the only finite set that has the uniform gaps property. The following is also clear.

Lemma 5.7. If $X, Y \subseteq \mathbb{Z}$ have the uniform gaps property. Then $X \cup Y$ has the uniform gaps property.

Lemma 5.8. If $C \subseteq \Gamma_{r}$ is a convex set, then $X=h^{-1}(C)$ has the uniform gaps property.

Proof. Let $C=(\alpha, \beta) \cap \Gamma_{r}$ and let $\gamma:=l(C)$. Take $m, n \in \mathbb{N}_{+}$such that $h(m)<\gamma^{1 / 2}$ and $h(-n)<\gamma^{1 / 2}$. We claim that $M=\max \{m, n\}$ witnesses that $X$ has the uniform gaps property.

Suppose that $x \in X$. If $h(x)<\alpha \gamma^{1 / 2}$, then $h(x) h(m) \in C$ and hence $x+m \in X$, and if $\alpha \gamma^{1 / 2}<h(x)$, then $h(x) h(n) \in C$ and hence $x+n \in$ $X$.

Remark. This proof does not work for larger models of $T_{r}^{*}$, because the length of $C$ might be infinitesimal with respect to $\Gamma_{r}$ and hence we cannot find suitable $m$ and $n$.

The following is Theorem 1.1 from the Introduction.

Theorem 5.9. Every infinite subset of $\mathbb{Z}$ definable in $\mathfrak{Z}$ has the uniform gaps property.

Proof. Let $X \subseteq \mathbb{Z}$ be definable in $\mathfrak{Z}$ and let $Y:=h(X)$.

By Corollary 5.5, there is $N>0$ such that $(h(n) Y)^{1 / N}$ is a finite union of convex sets and singletons for each $n \in\{0,1, \ldots, N-1\}$.

Using the second and the last part of Lemma 5.1

$$
Y=\bigcup_{n=0}^{N-1} h(-n)\left((h(n) Y)^{1 / N}\right)^{(N)}
$$

Therefore by Lemma 5.7, it suffices to show that $h^{-1}\left(\left((h(n) Y)^{1 / N}\right)^{(N)}\right)$ has the uniform gaps property for each $n$. Since $y \in\left((h(n) Y)^{1 / N}\right)^{(N)}$ if and only if $y=z^{N}$ for some $z \in(h(n) Y)^{1 / N}$, it suffices to show that 
$h^{-1}\left((h(n) Y)^{1 / N}\right)$ has the uniform gaps property. It is indeed the case using Lemmas 5.7 and 5.8 .

Corollary 5.10. Let $X$ be an infinite subset of $\mathbb{Z}$ that is definable in 3. Then there is $M \in \mathbb{N}_{+}$such that for every $x \in X$, the intersection $X \cap\{x-1, x-2, \ldots, x-M\}$ is nonempty.

Proof. The set $-X:=\{y \in \mathbb{Z}:-y \in X\}$ is also definable in $\mathfrak{Z}$ and hence has the uniform gaps property. That translates to $X$ as the conclusion of the corollary.

Corollary 5.11. Ordering of $\mathbb{Z}$ is not definable in $\mathfrak{Z}$.

Proof. If the ordering were definable in $\mathfrak{Z}$, then so would be the set of positive elements. However, $\mathbb{N}_{+}$does not satisfy the conclusion of Corollary 5.10.

Corollary 5.12. Multiplication is not definable in $\mathfrak{Z}$.

Proof. If multiplication on $\mathbb{Z}$ is definable, then the ordering of $\mathbb{Z}$ is also definable using Lagrange's four-square theorem.

We need the following lemma to show that $\mathfrak{Z}$ is unstable.

Lemma 5.13. Let $n>0$, then there is $m>0$ such that $m, 2 m, \ldots, n m \in$ $P_{r}$ and $-m,-2 m, \ldots,-n m \notin P_{r}$.

Proof. Let $\alpha=\min \{h(1), h(-1)\}$ and $\beta=\alpha^{\frac{1}{n+1}}$. By regular density of $\Gamma_{r}$ take $m \in \mathbb{N}_{+}$such that $\beta^{-1}<h(m)$. Clearly, this $m$ satisfies the conclusion of the lemma.

Proposition 5.14. The theory $T_{r}^{*}$ is not stable.

Proof. Let $\phi(x ; y)$ be the $L_{P}$-formula $y-x \in P$. We show that this formula is unstable. For this, it suffices to prove the following: For every $n>0$, there are $a_{1}, \ldots, a_{n} \in \mathbb{Z}$ such that

$$
\mathfrak{Z} \models \phi\left(a_{i}, a_{j}\right) \Longleftrightarrow i<j .
$$

So let $n>0$ be given. Take $a_{i}=i m$ where $m=m(n)$ is as in the lemma above. Now $a_{j}-a_{i}=(j-i) m$ and hence $\phi\left(a_{i}, a_{j}\right)$ holds in $\mathfrak{Z}$ if and only if $i<j$.

(We would like to thank Haydar Göral for the idea of this proof.)

\section{No REDUCTS}

We prove that there are no intermediate structures between $(\mathbb{Z},+)$ and $\left(\mathbb{Z},+, P_{r}\right)$; actually we prove the same result for $\left(\Gamma_{r}, \cdot\right)$ and $\left(\Gamma_{r}, \cdot, P_{r}\right)$.

We first consider a subset $X$ of $\Gamma_{r}$ that is definable in $\mathfrak{G}$. 
Proposition 6.1. Let $X \subseteq \Gamma_{r}$ be definable in $\mathfrak{G}$. Suppose that $X$ is not definable in $\left(\Gamma_{r}, \cdot\right)$. Then there is $N>0$ and $L \in\{0,1, \ldots, N-1\}$ such that both the set $(h(L) X)^{1 / N}$ and its complement contain a convex set.

Proof. By Corollary 5.5, there is $N>0$ such that for each $n$, the set $(h(n) X)^{1 / N}$ is a finite union of convex sets with end points in $\left\langle\Gamma_{r}\right\rangle$, and singletons. Write

$$
X=\bigcup_{n=0}^{N-1} h(-n)\left(h(n) X \cap \Gamma_{r}^{(N)}\right) .
$$

For each $n$, raising to power $N$ is a bijection between the sets $(h(n) X)^{1 / N}$ and $h(n) X \cap \Gamma_{r}^{(N)}$. Since $X$ is not definable in $\left(\Gamma_{r}, \cdot\right)$, there is $L \in$ $\{0, \ldots, N-1\}$ such that $(h(L) X)^{1 / N}$ is infinite. If $(h(L) X)^{1 / N}$ is cofinite in $\Gamma_{r}$, then so is $h(L) X \cap \Gamma_{r}^{(N)}$. Once again, since $X$ is not definable in $\left(\Gamma_{r}, \cdot\right), h(L) X \cap \Gamma_{r}^{(N)}$ cannot be cofinite in $\Gamma_{r}$. Therefore both $(h(L) X)^{1 / N}$ and its complement contains a convex set.

So if $N$ and $L$ are as in this proposition, both $(h(L) X)^{1 / N}$ and its complement are finite unions of convex sets and singletons, but neither is finite. Moreover, by Corollary 5.5, the end points of the convex sets appearing in either union are in $\left\langle\Gamma_{r}\right\rangle$. Therefore there is a finite union of convex sets with end points in $\left\langle\Gamma_{r}\right\rangle$ that is definable in $\left(\Gamma_{r}, \cdot, X\right)$ and is not cofinite in $\Gamma_{r}$.

Lemma 6.2. Let $X=(\alpha, \beta) \cap \Gamma_{r}^{(m)}$ and suppose that $\gamma:=\alpha \beta \in \Gamma_{r}$. Then a convex set is definable in $\left(\Gamma_{r}, \cdot, X\right)$. Moreover, the end points of that convex set are in $\left\langle\Gamma_{r} \cup\{\alpha, \beta\}\right\rangle$.

Proof. Let $0 \leq n<m$ be such that $\gamma \in h(n) \Gamma_{r}^{(m)}$.

We first consider the case that $n \neq 0$. Let $k:=\operatorname{gcd}(n, m), n^{\prime}=n / k$, and $m^{\prime}=m / k$. So $\gamma=h(n) \gamma_{0}^{m}=\left(h\left(n^{\prime}\right) \gamma_{0}^{m^{\prime}}\right)^{k}$ for some $\gamma_{0} \in \Gamma_{r}$.

Let $l \in \mathbb{Z}$ be such that $h\left(n^{\prime}\right) \gamma_{0}^{m^{\prime}} \in\left(\zeta_{k}^{l}, \zeta_{k}^{l+1}\right)$. Also let

$$
\delta_{0} \in\left(\left(\beta^{1 / k}\right)^{-1} \zeta_{k}^{-l},\left(\beta^{1 / k}\right)^{-1} \zeta_{k}^{-l+1}\right) \cap \gamma_{0}^{-m^{\prime}} \Gamma_{r}^{(m)} \text {, and } \delta=\delta_{0}^{k} .
$$

By Lemma [5.2, the set $Y:=(\alpha, \beta \delta) \cap \Gamma_{r}^{(m)}$ is definable in $\left(\Gamma_{r}, \cdot, X\right)$ and using Lemma 2.3 we have that $\gamma_{Y}:=\alpha^{1 / k}(\beta \delta)^{1 / k} \in h\left(n^{\prime}\right) \Gamma_{r}^{(m)}$. In particular, $\left\{1, \gamma_{Y}, \gamma_{Y}^{2}, \ldots, \gamma_{Y}^{m-1}\right\}$ is a full set of representatives of cosets of $\Gamma_{r}^{(m)}$ in $\Gamma_{r}$.

By Proposition 2.2

$$
Y^{1 / k}=\bigcup_{s=0}^{k-1} \zeta_{k}^{s}\left(\alpha^{1 / k},(\beta \delta)^{1 / k}\right) \cap \Gamma_{r}^{\left(m^{\prime}\right)} .
$$


Take $\tau_{1}, \tau_{2} \in \Gamma_{r}^{(m)}$ such that $\left(\alpha^{1 / k},(\beta \delta)^{1 / k}\right) \subseteq\left(\alpha \tau_{1}, \beta \tau_{2}\right)$ and $l\left(\left(\alpha \tau_{1}, \beta \tau_{2}\right)\right)<$ $\zeta_{k}$. The set $\left(\alpha \tau_{1}, \beta \tau_{2}\right) \cap \Gamma_{r}^{(m)}$ is definable in $\left(\Gamma_{r}, \cdot, X\right)$ by Lemma 5.2 and hence

$$
Z:=\left(\left(\alpha \tau_{1}, \beta \tau_{2}\right) \cap \Gamma_{r}^{(m)}\right) \cap Y^{1 / k}=\left(\alpha^{1 / k},(\beta \delta)^{1 / k}\right) \cap \Gamma_{r}^{(m)} .
$$

is also definable in $\left(\Gamma_{r}, \cdot, X\right)$.

Let $f: \Gamma_{r} \rightarrow \Gamma_{r}$ be defined as $f(x)=x^{-1} \gamma_{Y}$. It is clear that

$$
x \in\left(\alpha^{1 / k},(\beta \delta)^{1 / k}\right) \Longleftrightarrow f(x) \in\left(\alpha^{1 / k},(\beta \delta)^{1 / k}\right) .
$$

Therefore $f^{i}(Z)=\left(\alpha^{1 / k},(\beta \delta)^{1 / k}\right) \cap \gamma_{Y}^{i} \Gamma^{(m)}$ for $i=0,1, \ldots, m-1$. Hence the proper convex set

$$
\left(\alpha^{1 / k},(\beta \delta)^{1 / k}\right) \cap \Gamma_{r}=Z \cup f(Z) \cup \cdots \cup f^{m-1}(Z)
$$

is definable in $\left(\Gamma_{r}, \cdot, X\right)$.

Now let $n=0$ and take $k>0$ maximum such that $\gamma \in \Gamma_{r}^{\left(m^{k}\right)}$; say $\gamma=\gamma_{0}^{m^{k}}$ with $\gamma_{0} \in\left(\zeta_{m^{k}}^{l}, \zeta_{m^{k}}^{l+1}\right) \cap \Gamma_{r}$.

Let

$$
\delta_{0} \in\left(\left(\beta^{1 / m^{k}}\right)^{-1} \zeta_{m^{k}}^{-l},\left(\beta^{1 / m^{k}}\right)^{-1} \zeta_{m^{k}}^{-l+1}\right) \cap\left(\Gamma_{r} \backslash \gamma_{0}^{-1} \Gamma_{r}^{(m)}\right) \text { and } \delta:=\delta_{0}^{m^{k}} .
$$

As in the previous case, we have $Y=(\alpha, \beta \delta) \cap \Gamma_{r}^{(m)}$ is definable in $\left(\Gamma_{r}, \cdot, X\right)$ and

$$
Y^{1 / m^{k}}=\bigcup_{s=0}^{m^{k}-1} \zeta_{m^{k}}^{s}\left(\alpha^{1 / m^{k}},(\beta \delta)^{1 / m^{k}}\right) \cap \Gamma_{r} .
$$

Again, there are $\tau_{1}, \tau_{2} \in \Gamma^{(m)}$ such that

$$
Z:=\left(\left(\alpha \tau_{1}, \beta \tau_{2}\right) \cap \Gamma_{r}^{(m)}\right) \cap Y^{1 / m^{k}}=\left(\alpha^{1 / m^{k}},(\beta \delta)^{1 / m^{k}}\right) \cap \Gamma_{r}^{(m)} .
$$

Now we have $\gamma^{*}:=\alpha^{1 / m^{k}}(\beta \delta)^{1 / m^{k}}=\gamma_{0} \delta_{0}$ is not in $\Gamma_{r}^{(m)}$. Therefore using the previous case a proper convex set is definable in $\left(\Gamma_{r}, \cdot, Z\right)$, hence in $\left(\Gamma_{r}, \cdot, X\right)$.

It follows from the proof that the end points of the proper convex set are in $\left\langle\Gamma_{r} \cup\{\alpha, \beta\}\right\rangle$.

Lemma 6.3. Let $X=(\alpha, \beta) \cap \Gamma_{r}$ and $m>0$ with $l(\alpha, \beta)<\zeta_{m}$. Then for $0<k \leq m$ :

$$
\left\{x_{1} x_{2} \cdots x_{k}: x_{1}, x_{2}, \ldots, x_{k} \in X\right\}=\left(\alpha^{k}, \beta^{k}\right) \cap \Gamma_{r}
$$

Proof. Let

$$
Y_{k}:=\left\{x_{1} x_{2} \cdots x_{k}: x_{1}, x_{2}, \ldots, x_{k} \in X\right\} .
$$

It is clear from the assumptions that $Y_{k} \subseteq\left(\alpha^{k}, \beta^{k}\right) \cap \Gamma_{r}$. We prove equality by induction on $k$. 
The case $k=1$ is trivial, so let $k>1$ and suppose that $Y_{k-1}=$ $\left(\alpha^{k-1}, \beta^{k-1}\right) \cap \Gamma_{r}$. Then

$$
\begin{aligned}
Y_{k} & =\bigcup_{x_{k} \in X} Y_{k-1} x_{k} \\
& =\bigcup_{x_{k} \in X}\left(\left(\alpha^{k-1}, \beta^{k-1}\right) \cap \Gamma_{r}\right) x_{k} \\
& =\bigcup_{x_{k} \in X}\left(x_{k} \alpha^{k-1}, x_{k} \beta^{k-1}\right) \cap \Gamma_{r} .
\end{aligned}
$$

Clearly, the last union is a convex set. Since $\alpha^{k}$ and $\beta^{k}$ are limit points of $Y_{k}$ (in $\mathbb{S}$ ), we get

$$
Y_{k}=\left(\alpha^{k}, \beta^{k}\right) \cap \Gamma_{r}
$$

Corollary 6.4. Let $X=(\alpha, \beta) \cap \Gamma_{r}$, where $\alpha, \beta \in\left\langle\Gamma_{r}\right\rangle$. Then an interval is definable in $\left(\Gamma_{r}, \cdot, X\right)$.

Proof. Let $N \in \mathbb{N}_{+}$such that $\alpha^{N}, \beta^{N} \in \Gamma_{r}$, and take $\gamma \in \Gamma_{r}$ such that $l((\alpha, \beta \gamma))<\zeta_{N}$. Then $\left(\alpha^{N}, \beta^{N} \gamma^{N}\right) \cap \Gamma_{r}$ is an interval and is definable in $\left(\Gamma_{r}, \cdot, X\right)$ by Lemma 6.3.

Lemma 6.5. Let $X$ be a finite union of convex sets whose end points are in $\left\langle\Gamma_{r}\right\rangle$. If $X$ is not cofinite in $\Gamma_{r}$, then an interval is definable in $\left(\Gamma_{r}, \cdot, X\right)$.

Proof. After translating, we may assume that $1 \notin X$.

Let $X=\bigcup_{i=1}^{m} C_{i}$ where $C_{i}=\left(\alpha_{i}, \beta_{i}\right) \cap \Gamma_{r}$ with

$$
\alpha_{1}<\beta_{1} \leq \alpha_{2}<\beta_{2} \leq \cdots \leq \alpha_{m}<\beta_{m} .
$$

We prove the result by induction on $m$. If $m=1$, then there is nothing to do. So suppose $m>1$.

Put $\lambda_{j}=\beta_{j} \alpha_{j}^{-1}$ for all $j$, and $\delta_{j}=\alpha_{j+1} \beta_{j}^{-1}$ for $j=1, \ldots, m$, where $\alpha_{m+1}:=\alpha_{1}$.

Below we consider three cases and in each one we construct a convex set definable in $\left(\Gamma_{r}, \cdot, X\right)$. Moreover the end points of those convex sets will still be in $\left\langle\Gamma_{r}\right\rangle$. Hence by using Corollary 6.4, we will be done.

Case 1: The set $\left\{\delta_{1}, \ldots, \delta_{m}\right\}$ is not a singleton.

Let $\delta_{j_{0}}$ and $\delta_{j_{1}}$ be the two smallest elements of this set and let $\lambda=$ $\min \left\{\delta_{j_{1}} \delta_{j_{0}}^{-1}, \lambda_{1}, \ldots, \lambda_{m}\right\}$. Take $\gamma \in\left(\lambda^{1 / 2}, \lambda\right) \cap \Gamma_{r}$ and let $N>0$ be such that

$$
\gamma<\gamma^{2}<\cdots<\gamma^{N-1}<\delta_{j_{0}}<\gamma^{N}
$$

Note that $\gamma^{N}<\delta_{j_{1}}$ and hence the union

$$
Y:=X \cup \gamma X \cup \gamma^{2} X \cup \cdots \cup \gamma^{N} X
$$


is a union of at most $m-1$ convex sets, yet it has infinite complement in $\Gamma_{r}$. So by induction hypothesis, there is a nonempty proper convex subset $C$ of $\Gamma$ that is definable in $\left(\Gamma_{r}, \cdot, Y\right)$. Since $Y$ is definable in $\left(\Gamma_{r}, \cdot, X\right)$, the set $C$ is definable in $\left(\Gamma_{r}, \cdot, X\right)$.

Case 2: The set $\left\{\delta_{1}, \ldots, \delta_{m}\right\}$ is a singleton, but the set $\left\{\lambda_{1}, \ldots, \lambda_{m}\right\}$ is not a singleton.

The set $Z=\Gamma_{r} \backslash X$ is a set as in Case 1. So a nonempty proper convex subset of $\Gamma$ is definable in $\left(\Gamma_{r}, \cdot, Z\right)$, hence in $\left(\Gamma_{r}, \cdot, X\right)$.

Case 3: Both $\left\{\delta_{1}, \ldots, \delta_{m}\right\}$ and $\left\{\lambda_{1}, \ldots, \lambda_{m}\right\}$ are singletons.

Note that we need to have $\delta_{j} \lambda_{j}=\zeta_{m}$ for each $j$. Hence $\alpha_{j+1}=\zeta_{m} \alpha_{j}$ and $\beta_{j+1}=\zeta_{m} \beta_{j}$ for each $j=1, \ldots, m-1$. Therefore

$$
X=\left(\bigcup_{j=0}^{m-1} \zeta_{m}^{j}\left(\alpha_{1}, \beta_{1}\right)\right) \cap \Gamma_{r} .
$$

So $X^{(m)}=\left(\alpha_{1}^{m}, \beta_{1}^{m}\right) \cap \Gamma^{(m)}$. Therefore a convex set is definable in $\left(\Gamma_{r}, \cdot, X\right)$ by Lemma 6.2 .

Putting Proposition 6.1 and Lemma 6.5 together, we get the desired result for unary definable sets.

Proposition 6.6. Let $X \subseteq \Gamma_{r}$ be definable in $\mathfrak{G}$, but not in $\left(\Gamma_{r}, \cdot\right)$. Then $\left(\Gamma_{r}, \cdot, X\right)$ is interdefinable with $\mathfrak{G}$.

Now we handle the general case.

Theorem 6.7. Let $X \subseteq \Gamma_{r}^{n}$ be definable in $\mathfrak{G}$, but not in $\left(\Gamma_{r}, \cdot\right)$. Then $\left(\Gamma_{r}, \cdot, X\right)$ is interdefinable with $\mathfrak{G}$.

Proof. We proceed by induction on $n$. Proposition 6.6 serves as the case $n=1$. So let's assume that $n>1$ and that the result holds for $m<n$.

So let $X \subseteq \Gamma_{r}^{n}$ be definable in $\mathfrak{G}$, and take $N \in \mathbb{N}_{+}$such that for every $\vec{a} \in \mathbb{Z}^{n}$, the set $(h(\vec{a}) X)^{1 / N}$ is a union of an open set and finitely many sets contained in affine sets. Write

$$
X=\bigcup_{\vec{a} \in A} h(-\vec{a})\left(h(\vec{a}) X \cap\left(\Gamma_{r}^{(N)}\right)^{n}\right),
$$

where $A$ is a finite subset of $\mathbb{Z}^{n}$. It is easy to see that for each $\vec{a}$, the set $(h(\vec{a}) X)^{1 / N}$ has empty interior if and only if $h(\vec{a}) X \cap\left(\Gamma_{r}^{(N)}\right)^{n}$ is contained in a finite union of affine sets.

First suppose that $(h(\vec{a}) X)^{1 / N}$ has empty interior for each $\vec{a} \in A$. Then $(h(\vec{a}) X)^{1 / N}$ is a finite union of sets contained in an affine set and each of those sets is in bijection with a subset of $\Gamma_{r}^{d}$ for some $d<n$ via a projection. If all of those subsets of $\Gamma_{r}^{d}$ are definable in $\left(\Gamma_{r}, \cdot\right)$, then so is $(h(\vec{a}) X)^{1 / N}$. This cannot be correct for all $\vec{a}$, because $X$ is not 
definable in $\left(\Gamma_{r}, \cdot\right)$. Hence we obtain a subset of $\Gamma_{r}^{d}$ that is not definable in $\left(\Gamma_{r}, \cdot\right)$, but is definable in $\left(\Gamma_{r}, \cdot, X\right)$. So we obtain the result using the induction hypothesis.

So we assume that the set $A^{*}$ of $\vec{a} \in A$ such that $(h(\vec{a}) X)^{1 / N}$ has nonempty interior is nonempty. Hence the set

$$
X^{*}=X \bigcup_{\vec{a} \in A^{*}} h(-\vec{a})\left(h(\vec{a}) X \cap\left(\Gamma^{(N)}\right)^{n}\right)
$$

is definable in $\left(\Gamma_{r}, \cdot, X\right)$ and $\left(h(\vec{a}) X^{*}\right)^{1 / N}$ has nonempty interior for each $\vec{a} \in A^{*}$. Put $Y:=\Gamma_{r} \backslash X^{*}$. If $(h(\vec{a}) Y)^{1 / N}$ has empty interior for each $\vec{a} \in A^{*}$, then we may proceed as in the previous case. Therefore we may assume that $(h(\vec{a}) Y)^{1 / N}$ has nonempty interior for some $\vec{a} \in A^{*}$.

For $\vec{\gamma} \in \Gamma_{r}^{n-1}$, consider the sets

$$
\begin{gathered}
X_{\vec{\gamma}}^{*}:=\left\{\delta \in \Gamma_{r}:(\vec{\gamma}, \delta) \in\left(h(\vec{a}) X^{*}\right)^{1 / N}\right\}, \\
Y_{\vec{\gamma}}:=\left\{\delta \in \Gamma_{r}:(\vec{\gamma}, \delta) \in(h(\vec{a}) Y)^{1 / N}\right\}
\end{gathered}
$$

For each $\vec{\gamma}$, these sets are either empty or has nonempty interior. If there is $\vec{\gamma} \in \Gamma_{r}^{n-1}$ such that both $X_{\vec{\gamma}}^{*}$ and $Y_{\vec{\gamma}}$ have nonempty interior, then $X_{\vec{\gamma}}^{*}$ is not definable in $\left(\Gamma_{r}, \cdot\right)$, and we are done using the induction hypothesis. Otherwise for each $\vec{\gamma} \in \Gamma_{r}^{n-1}$, the set $X_{\vec{\gamma}}^{*}$ is either empty or is $\Gamma_{r}$. Then the set $Z:=\left\{\vec{\gamma}: X_{\vec{\gamma}}^{*}=\Gamma_{r}\right\}$ is a subset of $\Gamma_{r}^{n-1}$ definable in $\left(\Gamma_{r}, \cdot, X\right)$. If $Z$ is definable in $\left(\Gamma_{r}, \cdot\right)$, then so are $\left(h(\vec{a}) X^{*}\right)^{1 / N}$ and $X$. Therefore $Z$ is not definable in $\left(\Gamma_{r}, \cdot\right)$ and once again we are done by the induction hypothesis.

\section{REFERENCES}

[1] Jean-Paul Allouche and Jeffrey Shallit. Automatic sequences. Cambridge University Press, Cambridge, 2003. Theory, applications, generalizations.

[2] Gabriel Conant. Multiplicative structure in stable expansions of the group of integers. Illinois J. Math., 62(1-4):341-364, 2018.

[3] Gabriel Conant. There are no intermediate structures between the group of integers and Presburger arithmetic. J. Symb. Log., 83(1):187-207, 2018.

[4] Ayhan Günaydın. Model Theory of Fields with Multiplicative Groups. PhD thesis, University of Illinois at Urbana-Champaign, 2008.

[5] Jonathan Kirby. An invitation to model theory. Cambridge University Press, Cambridge, 2019.

[6] Quentin Lambotte and Françoise Point. On expansions of $(\mathbf{Z},+, 0)$. Ann. Pure Appl. Logic, 171(8):102809, 36, 2020.

[7] Daniel Palacín and Rizos Sklinos. On superstable expansions of free Abelian groups. Notre Dame J. Form. Log., 59(2):157-169, 2018.

[8] Bruno Poizat. Supergénérix. J. Algebra, 404:240-270, 2014. À la mémoire d'Éric Jaligot. [In memoriam Éric Jaligot].

[9] M.C. Tran and E. Walsberg. A family of $d_{p}$-minimal expansions of the additive group of integers. Preprint. 
Department of Mathematics, BoĞaziçi University, BebeK, Istanbul, TURKEY

Email address: ayhan.gunaydin@boun.edu.tr

Department of Mathematics, BoĞaziçı University, Bebek, Istanbul, TURKEY

Email address: assilem89@gmail.com 